\title{
Application of Polydioxanone Sutures in the Nuss Procedure
}

\author{
Yimin $X_{i e}^{1}$ Jinbo Ning ${ }^{2}$ \\ ${ }^{1}$ Department of Pediatric Surgery, Chongqing University Three \\ Gorges Hospital, Chongqing, China \\ 2 Department of Pediatric Surgery, Chongqing University Three \\ Gorges Hospital, Chongqing, China
}

Thorac Cardiovasc Surg 2022;70:77-82.

\begin{abstract}
Address for correspondence Jinbo Ning, MD, Three Gorges Hospital, Chongqing University, Chongqing, China

(e-mail: popining@outlook.com).
\end{abstract}

\begin{abstract}
\section{Keywords}

- pectus excavatum

- Nuss procedure

- minimally invasive repair of pectus excavatum (MIRPE)

- polydioxanone suture
\end{abstract}

Background/Purpose The Nuss procedure is the most common surgical repair for pectus excavatum (PE). Surgical steel wires are used in some modifications of the Nuss procedure to attach one or both ends of a support bar to the ribs. During follow-up, wire breakage was found in some cases. Patients with wire breakage may undergo prolonged bar removal surgery and may be exposed to excessive radiation.

In this study, we had a series of patients who received polydioxanone suture (PDS) fixations instead of steel wires. This retrospective study was conducted to explore the differences between these two fixation materials in the incidence of related complications and efficacies. Furthermore, we attempted to observe whether the two materials lead to similar surgical efficacy in the Nuss procedure, whether they have divergent effects on the bar removal surgery, and whether PDS can reduce the risks due to steel wire breakage as expected.

Methods We retrospectively studied PDS and surgical steel wires as fixation materials for the Nuss procedure in children with congenital PE and reviewed the outcomes and complications. A total of 75 children who had undergone Nuss procedure repairs and bar removals from January 2013 to December 2019 were recruited to participate in this study. They were divided into three groups: the PDS group, the unbroken wire (UBW) group, and the broken wire (BW) group, according to the fixation materials and whether the wires had broken or not. Moreover, we selected the duration of operation (DO), intraoperative blood loss (BL), bar displacement (BD), postoperative pain score (PPS), and incision infection as the risk indicators and the postrepair Haller index $(\mathrm{HI})$ as the effectiveness indicator. These indicators were statistically compared to determine whether there were differences among the three groups.

Results One BD occurred in the PDS and BW groups while none took place in the UBW group. No incision infection was found in any of the groups. The PDS group had the shortest DO, while the DO in the UBW group was shorter than that in the BW group $(p<0.05)$. BL in the PDS group was less than that in the other two groups $(p<0.05)$. received

August 28, 2020

accepted after revision

December 30, 2020

published online

February 18, 2021
DOI https://doi.org/ 10.1055/s-0041-1723847. ISSN 0171-6425.
(C) 2021. The Author(s).

This is an open access article published by Thieme under the terms of the Creative Commons Attribution-NonDerivative-NonCommercial-License, permitting copying and reproduction so long as the original work is given appropriate credit. Contents may not be used for commercial purposes, or adapted, remixed, transformed or built upon. (https://creativecommons.org/ licenses/by-nc-nd/4.0/)

Georg Thieme Verlag KG, Rüdigerstraße 14, 70469 Stuttgart, Germany 
Additionally, no difference was observed in BL between the BW and UBW groups $(p>0.05)$. The PPS of the PDS group was less than that of the BW group $(p<0.05)$, whereas no differences were found between the other two groups. No statistical difference emerged in $\mathrm{HI}$ among the groups $(p>0.05)$.

Conclusion PDS fixation results in a similar repair outcome and shows certain advantages in the DO, BL, and PPS; also, PDSs are safe and effective in the Nuss procedure.

Level of evidence Level III.

\section{Introduction}

A new, minimally invasive repair of pectus excavatum (MIRPE), or Nuss procedure, was first described by Dr. Donald Nuss in 1998. ${ }^{1}$ The Nuss procedure was quickly adopted by doctors and patients around the world because of its excellent outcomes, short procedural length, and outstanding cosmetic results. A series of clinical research and modifications has been conducted to improve the outcome and decrease related complications, including how to prebend the bar and whether to use thoracoscopy or a single stabilizer, two stabilizers, or even none and others. $^{2-4}$ In some modifications of the Nuss procedure, as in this study, surgical steel wires are employed to attach one or both ends of a support bar to the ribs with the use of stabilizer(s) or without. During follow-up, it was found that steel wires tended to break. The broken wire (BW) was difficult to remove when bar removal was performed for being tightly wrapped by new grown osseous tissues. Therefore, patients may experience excessive exposure to radiation and undergo prolonged surgery. Considerable research on pectus excavatum (PE) treatment has recently focused on how to improve the correction effects, but very few studies have investigated bar removal. In this study, we utilized polydioxanone suture (PDS) to replace wires in the Nuss procedure, which resulted in good correction outcomes and certain advantages in the following bar removal surgery.

\section{Methods}

\section{Patient Selection}

The records of patients with congenital PE who underwent the Nuss procedure and bar removal from January 2013 to
December 2019 were evaluated retrospectively. Children should meet two or more of the following criteria to be accepted for a MIRPE: (1) Haller index (HI) on computed tomography examination is greater than 3.25 ; (2) lung function examination suggests restrictive obstructive airway disease; (3) incomplete right bundle branch block on electrocardiography and Mitral valve prolapse on echocardiography; (4) the deformity progresses with obvious symptoms; and (5) psychosocial problems. After a bar dwell duration of 25 to 48 months, bar removal surgery was performed.

- Table 1 lists general information. Forty-two children (36 boys and 6 girls) accepted the \#0-PDS (Ethicon LLC, USA) fixation, and 33 children ( 28 boys and 5 girls) accepted the surgical steel wire fixation. Wire breakage was found in 14 cases. According to the fixation materials and whether a wire was broken or not, the patients were divided into three groups: the PDS group, the unbroken wire (UBW) group, and the BW group. Based on personal experience, we generally inserted the support bar from the left lateral chest. Only patients with one single support bar and one stabilizer on the left lateral chest wall were recruited to reduce the bias. Systemic diseases or malformations (such as severe congenital heart disease, digestive tract malformations, and other thoracic deformities) and other diseases that required concurrent surgeries were the exclusion criteria.

\section{Research Methods}

All the patients were routinely recorded for demographic characteristics. The HI and bar displacement (BD) or wire breakage were assessed by X-ray films during the follow-up. All the bar removal surgeries were performed according to a

Table 1 General information

\begin{tabular}{|c|c|c|c|c|}
\hline Group & PDS & UBW & BW & $p$-Value \\
\hline Male $(n)$ & 36 & 17 & 12 & \multirow[t]{2}{*}{ - } \\
\hline Female $(n)$ & 6 & 2 & 2 & \\
\hline Age $(y)$ & $8.5(7.5,12.1)$ & $8.3(7.3,10.7)$ & $10.6(8,13)$ & $0.315^{\mathrm{a}}$ \\
\hline Height $(\mathrm{cm})$ & $143(133,154)$ & $127(123,146)$ & $135(124,153)$ & $0.055^{\mathrm{a}}$ \\
\hline Weight $(\mathrm{kg})$ & $38(27,41)$ & $29(25,37)$ & $37(30,39)$ & $0.178^{\mathrm{a}}$ \\
\hline Haller index & $2.3(1.8,2.5)$ & $2.2(2.0,2.4)$ & $2.3(2.2,2.6)$ & $0.257^{\mathrm{a}}$ \\
\hline Bar dwell duration (month) & $36(33,40)$ & $36(33,45)$ & $43(36,47)$ & $0.09^{\mathrm{a}}$ \\
\hline
\end{tabular}

Abbreviations: BW, broken wire; PDS: polydioxanone suture; UBW, unbroken wire.

${ }^{\mathrm{a}}$ Kruskal-Wallis test. 
Table 2 Risk indicators and effectiveness indicator of the two groups

\begin{tabular}{|l|l|l|l|l|}
\hline Group & PDS & UBW & BW & -Value $^{\mathrm{a}}$ \\
\hline DO (min) & $\begin{array}{l}40 \\
(37,55)\end{array}$ & $\begin{array}{l}50 \\
(38,68)\end{array}$ & $\begin{array}{l}71 \\
(44,80)\end{array}$ & 0.00 \\
\hline BL $(\mathrm{mL})$ & $\begin{array}{l}11 \\
(5,9)\end{array}$ & $\begin{array}{l}16 \\
(9,16)\end{array}$ & 0.00 \\
\hline PPS & $3(2,3)$ & $3(2,3)$ & $3(3,4)$ & 0.00 \\
\hline $\mathrm{HI}$ & 2.3 & 2.2 & 2.3 & 0.20 \\
& $(1.8,2.5)$ & $(2.0,2.4)$ & $(2.2,2.6)$ & \\
\hline
\end{tabular}

Abbreviations: BL, blood loss; BW, broken wire; DO, duration of operation; HI, Haller index; PDS, polydioxanone suture; PPS, postoperative pain score; UBW, unbroken wire.

${ }^{a}$ Kruskal-Wallis test.

standard protocol by the same group of experienced surgeons under general anesthesia. Ibuprofen $\mathrm{Q} 6 \mathrm{H}$ at a weightadjusted dose was administered on the basis of a schedule until the end of postoperative day 2 . The postoperative pain severity was recorded using the Faces Pain Rating Scale, ${ }^{5}$ and a morphine prescription was provided for sharp pain when the postoperative pain score (PPS) was more than 3 . The duration of operation (DO) was accurately recorded, and blood loss (BL) was obtained by weighing gauze. A follow-up protocol with an X-ray test 6 months after bar removal was performed to measure the HI. ${ }^{6}$ Data on the DO, BL, BD, PPS, and postoperative incision infection were collected as risk indicators, and the $\mathrm{HI}$ was collected as the effectiveness indicator. Statistical analyses were conducted to observe whether there were statistical differences between the risk indicators and effectiveness indicator among groups. Due to the retrospective nature of this work, ethical approval was not required. Written informed consent was, however, obtained from all the participants and their parents.

\section{Statistical Analysis}

The statistical analyses were performed using SPSS Statistics, Version 22 (IBM, Armonk, NY). The data were expressed as median $\left(\mathrm{P}_{25}, \mathrm{P}_{75}\right)$, number, and percentage, and the categorical variables were compared using a chi-squared test or Fisher's exact test. The continuous variables were also compared using the Kruskal-Wallis test as the data were not normally distributed. Two-tailed $p$-values $<0.05$ were considered significant.

\section{Results}

- Table 2 presents the main results and - Fig. 1 depicts the Xray films of the two patients fixed with wire in BW group and UBW group separately. - Fig. 2 shows the intraoperative and postoperative pictures of a patient fixed with PDS, and the Xray films after MIRPE and bar removal.

There were two cases of two BDs in this series of patients. One BD was observed in the PDS group. The patient fell from a height and hit his lateral chest on the ground 25 months after MIRPE, necessitating early bar removal. Another patient in the BW group had a violent collision with a classmate 30 months following MIRPE and also accepted an early bar removal. No BD was found in the UBW group. No incision infections were found in any of the groups.

Among the three groups of cases, the DO of the PDS group was $40(37,55)$ minutes, which was the shortest, while the DO of the UBW group was $50(38,68)$ minutes. In contrast, the DO of the BW group was the longest, reaching 71 (44, 80) minutes; the Kruskal-Wallis test was performed on the DO of the three groups, and there were statistically significant differences $(p<0.05$; - Table 3 ).

The BL is the shortest in the PDS group, at $7(5,9) \mathrm{mL}$. The BL of the UBW group and the BW group were $11(7,14) \mathrm{mL}$ and $16(9,16) \mathrm{mL}$, respectively. The BL of the PDS group was lower than that of the other two groups $(p<0.05)$, but there was no statistically significant difference in the BL between the BW group and the UBW group ( $p>0.05 ;-$ Table 4$)$.

The PPS of the PDS group was $3(2,3)$, the PPS of the UBW group was $3(2,3)$, and the PPS of the BW group was $3(3,4)$. The PPS of the PDS group was lower than that of the BW group $(p<0.05)$, and there was no difference between the PDS and UBW groups or between the BW and UBW groups ( - Table 5 ).

The HI was obtained by an X-ray examination 6 months after bar removal. The $\mathrm{HI}$ of each group is, respectively: 2.3 $(1.8,2.5)$ for the PDS group, $2.2(2.0,2.4)$ for the UBW group, and $2.3(2.2,2.6)$ for the BW group. When the Kruskal-Wallis
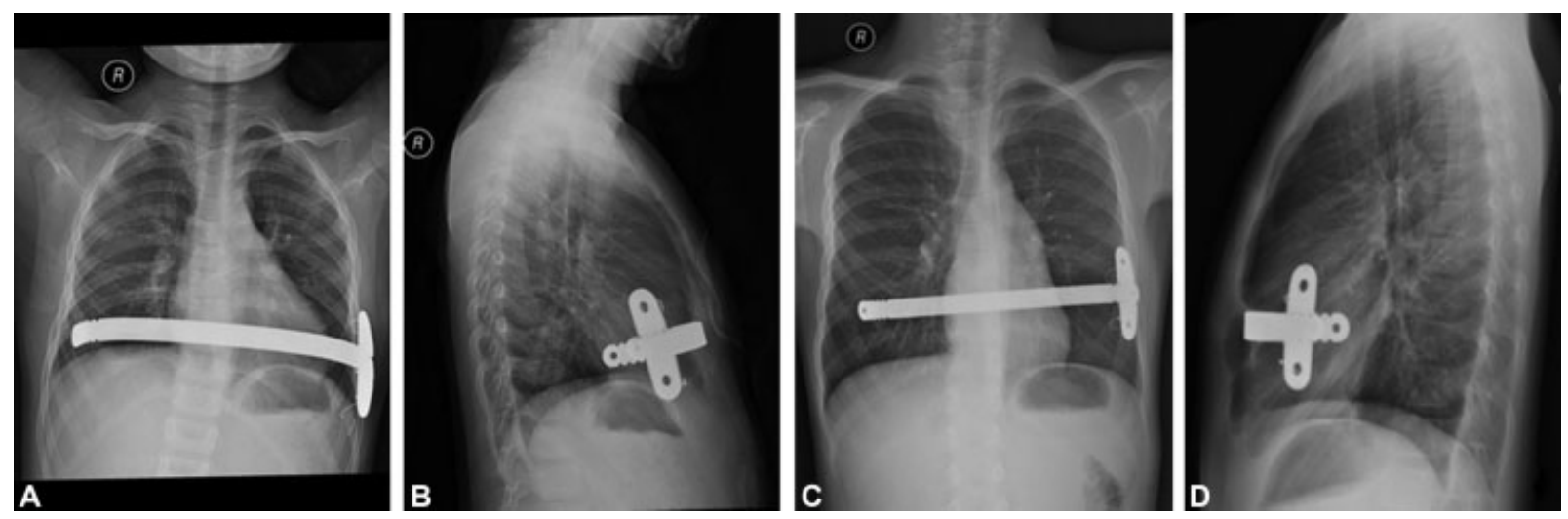

Fig. 1 (A, B) Posterior-anterior and right-lateral X-ray films with wire fixation 30 months after MIRPE in BW group. (C, D) Posterior-anterior and left-lateral X-ray films with wire fixation 34 months after MIRPE in UBW group. BW, broken wire; MIRPE, minimally invasive repair of pectus excavatum; UBW, unbroken wire. 

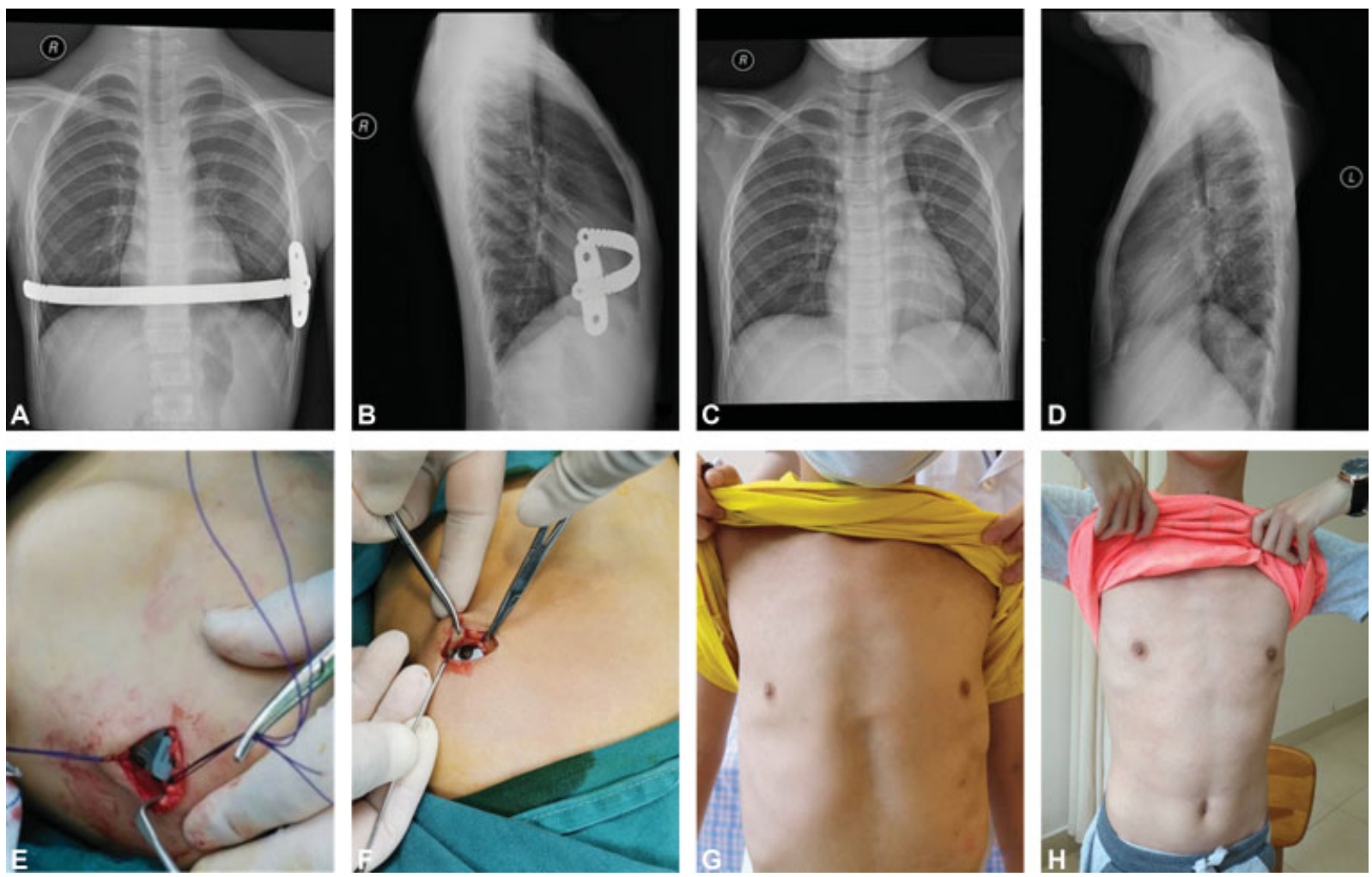

Fig. 2 (A, B) Posterior-anterior and right-lateral X-ray films with PDS fixation 36 months after MIRPE. (C, D) Posterior-anterior and right-lateral Xray films with PDS fixation 6 months after bar removal. (E) Fixing the stabilizer with circumcostal PDS fixation. (F) Cutting the soft tissue in the hole of stabilizer with a small needle-knife; no wire needs to be removed. (G) Postoperative frontal view 6 months after MIRPE. (H) Postoperative frontal view 6 months after bar removal. MIRPE, minimally invasive repair of pectus excavatum; PDS, polydioxanone suture.

Table 3 Pairwise comparisons of DO group

\begin{tabular}{|l|l|l|l|l|}
\hline Group & $\begin{array}{l}\text { Test } \\
\text { statistic }\end{array}$ & $\begin{array}{l}\text { Standard } \\
\text { error }\end{array}$ & $\begin{array}{l}\text { Standard } \\
\text { test statistic }\end{array}$ & $p$-Value \\
\hline PDS-UBW & -18.04 & 6.02 & -3.00 & 0.01 \\
\hline PDS-BW & -39.04 & 6.72 & -5.81 & 0.00 \\
\hline UBW-BW & -20.99 & 7.67 & -2.74 & 0.02 \\
\hline
\end{tabular}

Abbreviations: BW, broken wire; DO, duration of operation; PDS, polydioxanone suture; UBW, unbroken wire.

Table 4 Pairwise comparisons of BL group

\begin{tabular}{|l|l|l|l|l|}
\hline Group & $\begin{array}{l}\text { Test } \\
\text { statistic }\end{array}$ & $\begin{array}{l}\text { Standard } \\
\text { error }\end{array}$ & $\begin{array}{l}\text { Standard } \\
\text { test statistic }\end{array}$ & $p$-Value \\
\hline PDS-UBW & -19.04 & 5.98 & -3.19 & 0.00 \\
\hline PDS-BW & -36.27 & 6.67 & -5.14 & 0.00 \\
\hline UBW-BW & -17.23 & 7.61 & -2.26 & 0.07 \\
\hline
\end{tabular}

Abbreviations: BL, blood loss; BW, broken wire; PDS, polydioxanone suture; UBW, unbroken wire.

test was performed, there was no statistically significant difference in HI among the three groups $(p>0.05)$.

\section{Discussion}

PE accounts for nearly 90\% of all congenital chest wall deformities and occurs in as many as 1 of every 300 to 400
Table 5 Pairwise comparisons of PPS group

\begin{tabular}{|l|l|l|l|l|}
\hline Group & $\begin{array}{l}\text { Test } \\
\text { statistic }\end{array}$ & $\begin{array}{l}\text { Standard } \\
\text { error }\end{array}$ & $\begin{array}{l}\text { Standard } \\
\text { test statistic }\end{array}$ & $p$-Value \\
\hline PDS-UBW & -10.25 & 5.29 & -1.94 & 0.16 \\
\hline PDS-BW & -22.82 & 5.91 & -3.86 & 0.00 \\
\hline UBW-BW & -12.57 & 6.74 & -1.86 & 0.19 \\
\hline
\end{tabular}

Abbreviations: BW, broken wire; PDS, polydioxanone suture; PPS, postoperative pain score; UBW, unbroken wire.

live births. ${ }^{7}$ Patients with PE suffer varying degrees of psychosocial and physiologic consequences due to malformations, such as impaired social development and pulmonary and/or cardiac dysfunctions. The MIRPE has become the most popular procedure due to advantages, including its excellent outcomes, short procedural length, and outstanding cosmetic results. ${ }^{8}$

Bar flipping is the most frequent complication of the MIRPE procedure, as reported in the literature, and results in recurrence and reoperation. ${ }^{9,10}$ For this reason, the proper stabilization of the pectus bar was considered one of the most important points for a good outcome. Researchers proposed different techniques to reduce bar flipping and displacement, including Hebra's "third point of fixation," 11 Castellani's lateral stabilizer technique, ${ }^{12}$ and double-bar insertion in adults with a severe PE reported by Yoon. ${ }^{13}$ Nuss et al attached lateral stabilizers to the muscle fascia to prevent BD in children. ${ }^{1}$ However, stabilizers fixed to the 
muscles can cause discomfort, restrict mobilization of the serratus or pectoralis muscle, or cause other local complications. Therefore, some researchers used steel wires to fix the stabilizers to the ribs instead of fixing them to the muscles. This, however, resulted in a new problem of wire breakage caused by circumcostal fixation with steel wire. In our series, we had a wire breakage rate of $42.4 \%$ in circumcostal wire fixation. The average age of the patients in the BW group was $10.6(8,13)$ years, while that of the UBW group was 8.3 (7.3, 10.7 ) years. Wires were more likely to break at a greater age. This may be due to a higher lever force against the wire in older children. Castellani et al reported a case of wire breakage and the piercing of lung tissue by a free edge of the BW, which caused a hemopneumothorax. ${ }^{12}$

In this study, although wire breakage occurred in 14 cases at a $42.4 \%$ rate, no apparent BD was found in patients with wire fixation, except one in the BW group. We speculated that the reverse force on the bar and stabilizer exerted by the sternum and ribs, with the addition of the wrap of the surrounding soft tissues, is enough to maintain the mechanical stability and keep the support bar in a stable position following breakage of the wire.

Given the risks and complications that may occur and the fact that the support bar can still maintain the right position after the wire breakage, an absorbable and reliable suture may be a good remedy to provide effective fixation before the tissue of the surgical site heals. We chose absorbable no. 0PDS-II (Ethicon LLC, Somerville, New Jersey, United States) as the fixation material.

The PDS first used in surgery in 1982 was made of poly(pdioxanone) polyesters. ${ }^{9}$ PDSs undergo biodegradation via hydrolysis, and the degradation metabolites are excreted, primarily in the urine. ${ }^{14}$ PDSs have been widely used in soft tissue repair, abdomen closure, tendon anastomosis, and closure of the sternum, showing certain advantages in terms of suture effectiveness and infection prevention. ${ }^{15-18}$ Clinical application and in vitro studies have confirmed that PDS can maintain effective strength retention in these surgical procedures. $^{19}$ Among the commonly used absorbable sutures, PDS can maintain higher stress and tension at the edges of tissue. ${ }^{20}$ In the follow-up, we found that wire breakage commonly occurred from 2 to 6 months after the MIRPE. The effective fixation of PDS can take up to 90 days, which is enough time for the soft tissue at the operation region to repair and ensure that the support bar has been effectively fixed. PDSs are absorbed within 180 to 210 days, ${ }^{21}$ while the time for bar removal is generally 2 to 4 years after the Nuss procedure. Thus there is no need to be concerned about the removal of PDS during the bar removal surgery.

We performed MIRPE as first described by Nuss, with one notable difference. ${ }^{1}$ In our procedure, we inserted the left side of the bar into the slot of the stabilizer, and then tied the stabilizer to the ribs with two circumcostal wires, or absorbable sutures, to prevent the bar from rotating. Because we did not change the specific steps of the surgical process in the primary MIRPE procedure, whether we used steel wire or PDS would have had no significant impact on operation time, $\mathrm{BL}$, or other surgery-related indicators.
After MIRPE, we implemented a series of routine X-ray examinations during follow-up to observe the bar position and measure the HI index. We found BD in both PDS and BW group, with a total bar dislocation rate of $2.7 \%$. In both casesthe patient in the PDS group who had fallen and hit his lateral chest on the ground and patient in the BW group who had a violent collision with a classmate-the BDs were due to a violent impact on the chest. Del Frari and Schwabegger reported a bar dislocation rate of $2.2 \%$ and introduced their circumcostal PDS technique, fixing the bar wings with circumcostal double-armed 0-PDS using a Deschamps needle bilaterally at the thoracic wall. ${ }^{22}$ Although their fixing method is slightly different from ours, both studies showed that the use of circumcostal PDS fixation resulted in a low BD rate.

We selected the $\mathrm{HI}$ as the effectiveness indicator. No statistical difference in the effectiveness indicator among the three groups was found postoperatively, showing that the patients with PDS fixation also obtained the same correction effect as those with steel wire fixation.

When performing bar removal in patients with steel wire fixation, the surgical procedure for finding and removing the wire may generally take more time. In this study, the DO of the patients with wire fixation, especially with wire breakage, was longer than that of the PDS group $(p<0.05)$. Although most wire residues may not harm the physical health of patients, both the patients and their guardians commonly have a strong desire to have the wires completely removed. We found that the steel wire tended to break into two or three parts. The wire segments firmly embedded in the newly grown osteotylus were extremely difficult to identify during the operation. Rib periosteum and osteotylus had to be stripped to remove the BWs. The free tip of the wire segment, when wrapped tightly by the callus, can pierce the lung, ${ }^{12}$ while other parts of the BW can migrate with physical activity. We cannot deny the possibility that BWs travel subcutaneously and may even cause cardiopulmonary or intercostal vessel damage. In addition, wire breakage will take more time and wider dissection to find and remove the segments during a bar removal surgery, potentially leading to greater damage and excessive exposure to radiation during the prolonged duration of the operation.

This study validated that BL in patients with PDS fixations was less than that in patients with wire fixations. Excessive dissection may cause an increased BL, and the PPS in the BW group was higher than that in other groups. We speculate that the main reasons were the considerably longer operation time and more surgical damage to the chest wall muscles and ribs.

We also had a follow-up protocol with an X-ray test 6 months after bar removal. No relapse has been found to date, but longer-term observation is still needed. We did not encounter hypesthesia in the presternal region related to the circumcostal fixation. When placing the circumcostal sutures or wires, we increased the curvature of the needle, which can make the needle tip close to the ribs and bypass the ribs. Perhaps this technique can allow the wire or suture to run between the rib and the nerve-vessel bundle, thereby avoiding subcostal nerve compression. 
In conclusion, the study findings have shown that PDS fixation is equally effective as the surgical steel wires used in the Nuss procedure. Additionally, it reduces the DO, BL, and the severity of postoperative pain. PDS can be used as a safe and effectual fixation material in the Nuss procedure. This was a retrospective study, and the absorbable feature of PDS might make the parents (guardians) show certain preference in selection. Therefore, further randomized studies are needed to reduce selection bias.

\section{Conflict of Interest}

None declared.

\section{References}

1 Nuss D, Kelly RE Jr, Croitoru DP, Katz ME. A 10-year review of a minimally invasive technique for the correction of pectus excavatum. J Pediatr Surg 1998;33(04):545-552

2 Bodenstein L, Notrica DM. Use of a capture-guidance surgical instrument to minimize muscle stripping during Nuss repair of pectus excavatum. J Laparoendosc Adv Surg Tech A 2019;29(06): 865-868

3 de Campos JR, Das-Neves-Pereira JC, Lopes KM, Jatene FB. Technical modifications in stabilisers and in bar removal in the Nuss procedure. Eur J Cardiothorac Surg 2009;36(02):410-412

4 Li G, Jiang Z, Xiao H, et al. A novel modified Nuss procedure for pectus excavatum: a new steel bar. Ann Thorac Surg 2015;99(05): 1788-1792

5 Wong DL, Baker CM. Pain in children: comparison of assessment scales. Pediatr Nurs 1988;14(01):9-17

6 Khanna G, Jaju A, Don S, Keys T, Hildebolt CF. Comparison of Haller index values calculated with chest radiographs versus $\mathrm{CT}$ for pectus excavatum evaluation. Pediatr Radiol 2010;40(11):1763-1767

7 Hebra A, Calder BW, Lesher A. Minimally invasive repair of pectus excavatum. J Vis Surg 2016;2:73

8 Kanagaratnam A, Phan S, Tchantchaleishvili V, Phan K. Ravitch versus Nuss procedure for pectus excavatum: systematic review and meta-analysis. Ann Cardiothorac Surg 2016;5(05):409-421

9 Nuss D, Croitoru DP, Kelly RE Jr, Goretsky MJ, Nuss KJ, Gustin TS. Review and discussion of the complications of minimally invasive pectus excavatum repair. Eur J Pediatr Surg 2002;12(04):230-234
10 Park HJ, Lee SY, Lee CS. Complications associated with the Nuss procedure: analysis of risk factors and suggested measures for prevention of complications. J Pediatr Surg 2004;39(03):391-395 , discussion 391-395

11 Hebra A, Gauderer MW, Tagge EP, Adamson WT, Othersen HB Jr. A simple technique for preventing bar displacement with the Nuss repair of pectus excavatum. J Pediatr Surg 2001;36(08): 1266-1268

12 Castellani C, Schalamon J, Saxena AK, Höellwarth ME. Early complications of the Nuss procedure for pectus excavatum: a prospective study. Pediatr Surg Int 2008;24(06):659-666

13 Yoon YS, Kim HK, Choi YS, Kim K, Shim YM, Kim J. A modified Nuss procedure for late adolescent and adult pectus excavatum. World J Surg 2010;34(07):1475-1480

14 Boland ED, Coleman BD, Barnes CP, Simpson DG, Wnek GE, Bowlin GL. Electrospinning polydioxanone for biomedical applications. Acta Biomater 2005;1(01):115-123

15 Bigdelian H, Sedighi M. Evaluation of sternal closure with absorbable polydioxanone sutures in children. J Cardiovasc Thorac Res 2014;6(01):57-59

16 Gupta D, Sharma U, Chauhan S, Sahu SA. Improved outcomes of scar revision with the use of polydioxanone suture in comparison to polyglactin 910: a randomized controlled trial. J Plast Reconstr Aesthet Surg 2018;71(08):1159-1163

17 Jordan MC, Boelch S, Jansen H, Meffert RH, Hoelscher-Doht S. Does plastic suture deformation induce gapping after tendon repair? A biomechanical comparison of different suture materials. J Biomech 2016;49(13):2607-2612

$18 \mathrm{Naz}$ S, Memon SA, Jamali MA, et al. Polydioxanone versus polypropylene closure for midline abdominal incisions. JAMC 2017;29 (04):591-594

19 Müller DA, Snedeker JG, Meyer DC. Two-month longitudinal study of mechanical properties of absorbable sutures used in orthopedic surgery. J Orthop Surg Res 2016;11(01):111

20 Liber-Kneć A, tagan S. The stress relaxation process in sutures tied with a surgeon's knot in a simulated biological environment. Polim Med 2016;46(02):111-116

21 Gierek M, Kuśnierz K, Lampe P, et al. Absorbable sutures in general surgery - review, available materials, and optimum choices. Pol Przegl Chir 2018;90(02):34-37

22 Del Frari B, Schwabegger AH. How to avoid pectus bar dislocation in the MIRPE or MOVARPE technique: results of 12 years' experience. Ann Plast Surg 2014;72(01):75-79 